\title{
Treatment of Acute Respiratory Distress Syndrome with Vasoactive Intestinal Peptide
}

Jihad G. Youssef, MD ${ }^{1,2}$

Sami Said, $\mathrm{MD}^{3}$

George Youssef, $\mathrm{BS}^{4}$

Matthew J. Javitt, MD ${ }^{5}$

Jonathan C. Javitt, MD, MPH ${ }^{6,7}$

1. Houston Methodist JC Walter Jr Transplant Center, Houston Methodist Research Institute, Houston Methodist Hospital, Houston, Texas, USA

2. Institute of Academic Medicine, Department of Medicine, Weill Cornell Medicine

3. Posthumous. At the time of this research, author SS was Professor of Medicine at State University of New York Health Sciences Center, Stony Brook, NY.

4. University of Houston, College of Natural Sciences and Mathematics

5. NYU Langone Medical Center, Brooklyn, NY

6. NeuroRx, Inc., Wilmington, DE

7. Johns Hopkins University School of Medicine, Baltimore, MD

Correspondence: jjavitt@jhu.edu

Disclosures: Authors JCJ and MJJ have a financial relationship to NeuroRx, Inc., the sponsor of RLF-100 (aviptadil) for treatment of COVID-19 and to Relief Therapeutics Holdings, AG, which owns intellectual property related to aviptadil. Author JGY has received funding as an investigator for RLF-100 through his institution.

Keywords: ARDS, VIP, Aviptadil, RLF-100, Acute Respiratory Distress Syndrome, Sepsis 


\begin{abstract}
Purpose: To assess the clinical safety and possible effectiveness of Vasoactive Intestinal Peptide in the treatment of Acute Respiratory Distress Syndrome (ARDS) related to sepsis

Methods: Under FDA Investigational New Drug clearance, 8 patients with ARDS related to sepsis were treated with $50 \mathrm{pmole} / \mathrm{kg} / \mathrm{hr}-100 \mathrm{pmole} / \mathrm{kg} / \mathrm{hr}$ of Vasoactive Intestinal Peptide by intravenous infusion for 12 hours. All patients were on mechanical ventilation and full telemetery.

Results: No drug-related serious adverse events were seen. Hypotension was seen in association with two infusions and diarrhea in association with one, but did not necessitate cessation of therapy. Bigeminy was seen in association with one infusion without sequelae. Seven of eight patients demonstrated a successful course during intensive care and were successfully removed from mechanical ventilation and discharged from intensive care. The eighth patient succumbed to purulent secretions in the lungs. Of those who were discharged from the ICU, six demonstrated successful 30 day survival. The seventh died from a cerebral infract at day 30, deemed unrelated to treatment with VIP. Serum levels of Tumor Necrosis Factor $\alpha$ were obtained in 6 patients at baseline and 24 hours and were seen to decrease with treatment in five patients.
\end{abstract}

Conclusions: Initial clinical results of treatment with VIP in patients with ARDS demonstrated a safety profile consistent with previous studies in normal volunteers. The successful clinical course seen in 7 of 8 patients in the setting of an expected 50\% survival may suggest that VIP shows promise in the treatment of other infectious conditions that damage the pulmonary epithelium, particularly COVID- 19.

\title{
Introduction
}

In March 2020, the US Food and Drug Administration cleared the investigational use of RLF-100 (aviptadil: a synthetic form of Vasoactive Intestinal Peptide (VIP)) for the treatment of Critical COVID19 with Respiratory Failure. Among the evidence of human safety included in the Investigational New Drug (IND) application was a previously unpublished study of patients with Acute respiratory distress syndrome (ARDS) in the setting of sepsis. RLF-100 has subsequently been granted a Fast Track Designation by FDA and initial clinical results in the treatment of Critical COVID-19 with Respiratory Failure will shortly be reported. This manuscript reports that prior clinical experience with the use of VIP in ARDS. The results were previously unreported owing to the retirement and subsequent demise of the senior author (SS). However, the clinical course of the treated patients was witnessed by author JGY, who was the primary treating physician for the study.

ARDS is a severe, diffuse inflammatory pulmonary disease with myriad etiologies, for which there is no approved therapy. The underlying condition causes a massive inflammatory response and diffuse edema throughout the lung, culminating in hypoxemia and respiratory distress or failure. Recent mortality estimates for ARDS range from 35-45\%, depending on disease severity, with the only accepted therapy being mechanical ventilation. ${ }^{1}$

Vasoactive intestinal peptide (VIP) is a ubiquitous neuropeptide throughout the human body. Since its discovery by Said et al. in 1970, (Said 1970), VIP has been identified in numerous major organ systems throughout the body.(Power 1988, Onoue 2004, Busto 2000, Feliciano 1998, Fracarroli 2012) but is $70 \%$ concentrated in the lung (Virgolini 1995). Within the lung, VIP receptors have primarily been identified on Alveolar Type II cells, which are critical to surfactant production (Li 2004) and recycling (Nicholas 1996). VIP also shown to reduce inflammatory cytokines. In vitro and in vivo studies using VIP have demonstrated an immunomodulatory effect, particularly in the setting of acute lung injury. In 
animal models, VIP downregulates numerous macrophage-mediated inflammatory cytokines as well as known proinflammatory receptors.(Delgado 1999, Ran 2015, Ganea 2015, Hernanz 1996, Ke 2017, Martinez 1998) Moreover, VIP is associated with an increased concentration of anti-inflammatory cytokine IL-10 and promotes the differentiation of Th2 cells in place of proinflammatory T-cell lines.(Wang et al. 2000).

In light of these results, VIP was proposed as a potential treatment for ARDS in 1996, but was not synthesized in sufficient quantity for study under FDA Investigational New Drug (IND) permission until 2005. The IND documents prior published and unpublished safety results in normal volunteers without serious adverse events. This manuscript reports results in the first eight patients treated with intravenous VIP under investigator-sponsored IND 52,088 issued to author SS. These results have not previously been reported in the peer-reviewed literature but were reported to and reviewed by FDA in connection with this and subsequent regulatory submissions. The objective of this investigator-sponsored study was to obtain preliminary data on the safety and efficacy of intravenous (IV) infused Aviptadil in patients with acute respiratory distress syndrome (ARDS) associated with sepsis.

The study originally anticipated recruitment of 16 patients but was terminated after 8 patients as a function of the retirement of the first author. These results are being published now, after 15 years because of renewed interest in the use of VIP to treat pneumonitis and lung injury associated with COVID-19. The principal investigator of this study (author SIS) is unfortunately deceased. However, the first author of this publication oversaw the clinical care of the patients in this study.

\section{Methods}

Human subjects were treated pursuant to IND 52,088 under a protocol approved by the Institutional Review Board of State University of New York Health Sciences Center, Stony Brook, NY. Patients who met the consensus criteria for diagnosis ARDS in the setting of sepsis were considered for this trial. Patients were observed for a 24-h period, during which time all inclusion criteria had to be met. The primary inclusion criteria were sepsis or septic shock, diagnosed ARDS, hypotension, and end organ disfunction. If all criteria had been met once (not necessarily simultaneously), the patient was enrolled and received the study drug over a 12 -hour period.

The eligibility criteria for the study were:

(1) ARDS. Acute respiratory failure characterized by: a) hypoxemia, refractory to supplemental $\mathrm{O}_{2}$ therapy; b) diffuse pulmonary infiltrates; c) absence of a cardiogenic cause of pulmonary edema; and d) reduced pulmonary compliance. The severity of lung injury in these patients is quantified by the scoring system of Murray (Murray 1988).

(2) diagnosis of Sepsis/septic shock, characterized by two or more of the following:

a. fever or hypothermia (core temp. $>38^{\circ} \mathrm{C}$ or $\left\langle 36^{\circ} \mathrm{C}\right.$ ); tachycardia (heart rate $>90$, in the absence of $\mathrm{B}$-adrenergic receptor blockade); tachypnea (respiratory rate $>20$ ) or $\mathrm{PaCO}_{2}<32 \mathrm{~mm} \mathrm{Hg}$ or requirement of mechanical ventilation; white blood cell count $>12,000$ cells $/ \mathrm{mm}^{3}$ or $<4,000$ cells $/ \mathrm{mm}^{3}$ or immature neutrophils (bands $>$ $10 \%)$;

b. Hypotension (systolic blood pressure $90 \mathrm{~mm} \mathrm{Hg}$, mean arterial blood pressure < $70 \mathrm{~mm} \mathrm{Hg}$, or sustained systolic blood pressure decrease of $40 \mathrm{~mm} \mathrm{Hg}$, unresponsive to fluid challenge, or the use of vasopressor agents (excluding dopamine at $<5.0 \mathrm{mg} / \mathrm{kg}$ per $\mathrm{min}$ ); 
c. Evidence of inadequate organ perfusion or organ dysfunction: acute deterioration in mental acuity (excluding edative drugs or other nonseptic causes of altered mental status); or unexplained metabolic acidosis $(\mathrm{pH}<7.30$ or elevated plasma lactate or base deficit of $>5 \mathrm{mEq} / \mathrm{L})$; or oliguria $(<0.5 \mathrm{ml} / \mathrm{kg} / \mathrm{hr})$ for $>2 \mathrm{~h}$; or unexplained coagulopathy (elevated PT or PTT or platelet count decreased to less than half the baseline value within the past $24 \mathrm{~h}$ or $<100,000 / \mathrm{mm}^{3}$; or an acute elevation of bilirubin to $>2.0 \mathrm{mg} / \mathrm{di}$ and an elevation in one of the liver enzymes (alkaline phosphatase, SGOT, SGPT).

d. Clinical suspicion of infection (to be documented by appropriate and accepted measures such as sputum Gram stain, urinalysis). Documented infections are defined as positive bacterial cultures from normally sterile body fluids, or Gram stain and positive cultures together with clinical correlation when sepsis originates from respiratory or genitourinary infections.

Exclusion criteria were as follows: Pregnancy; < 18 years old; irreversible underlying condition with rapidly fatal course; current or recent (within $30 \mathrm{~d}$ ) enrollment in another investigational trial; severe burns or uncontrolled hemorrhage (4-unit transfusion requirement in previous $24 \mathrm{~h}$ ); acquired immune deficiency syndrome; transplant patients currently immunosuppressed; chemotherapy-induced neutropenia (granulocyte count $<1000 / \mathrm{mm} 3$ ); weight $>100 \mathrm{~kg}$; cardiogenic shock; anuria (urine output $<50 \mathrm{ml} / \mathrm{d}$ ); severe liver disease with portal hypertension; recent stroke, head trauma, increased intracranial pressure, or other serious neurologic disorder; inability to obtain informed consent or assent.

\section{Study drug:}

\section{Preparation and Delivery:}

Sterile, pyrogen free VIP is prepared, as in earlier human studies, at the Karolinska Institute, Stockholm, Sweden (where it was first isolated by the Pl in collaboration with Prof. Viktor Mutt) and shipped to New York in sealed ampules or vials. Stock solutions of the peptide are kept at $-70^{\circ} \mathrm{C}$ in the hospital pharmacy, under the direct care of the senior pharmacist in charge of the investigational drug service at University Hospital. Solutions for i.v. infusion were prepared hours before use, in sterile isotonic saline, containing $0.5 \%$ human albumin for each individual patient, by the pharmacist, and delivered by a volumetric infusion pump.

\section{Results}

Safety. The objective of this Phase I study was to obtain preliminary data, in an open-label study, on the safety of Aviptadil, infused iv. (50 or $100 \mathrm{pmol} / \mathrm{kg} / \mathrm{hr}$, infusion time 6 to $12 \mathrm{hr}$ ) in patients with ARDS induced by sepsis. In total, 8 patients were included in the study. Three patients progressed to the high dose group while infusion in the remaining 5 patients was maintained at the lower dose. In 5 cases, there was surgery preceding the infection. Consistent with the polymorbidity of the patients admitted to the trial, they also received a large number of concomitant medications and other supportive measures.

In the lower dose group, Aviptadil administration was stopped in one (bronchial obstruction due to hypersecretion considered to be unrelated to Aviptadil) and the dose halved in another (Hypotension). In the high dose group with $100 \mathrm{pmol} / \mathrm{kg} / \mathrm{hr}$ (3 patients) Aviptadil administration was stopped in none. However, dose was transitorily reduced in two, due to hypotension (1 case) or to bigeminy (1 case). Watery stools were observed in one patient in the high dose group. 
Two patients died during the long-term follow-up period. Patient \# 5, aged 87 years, male, suffered was successfully extubated and discharged from the ICU, but succumbed to a massive right-sided middle cerebral artery infarct three weeks after VIP administration. Supportive measures were stopped at the request of the family. Patient \# 2, aged 80 years, male, received the Aviptadil infusion for only 2 of the intended 6 hours because oxygen saturation dropped to $85 \%$. The patient at this point was suctioned from the endotracheal tube and ventilated with an AMBU bag + bronchodilator nebulizer treatment. The endotracheal tube yielded large amount of thick, purulent secretions. The arterial oxygen saturations improved to baseline of $90-92 \%$, but the Aviptadil infusion was not restarted. He died 4 days later; the death was considered a direct consequence of underlying sepsis and unrelated to VIP infusion.

\section{Figure 1. VIP dosing per patient over time}

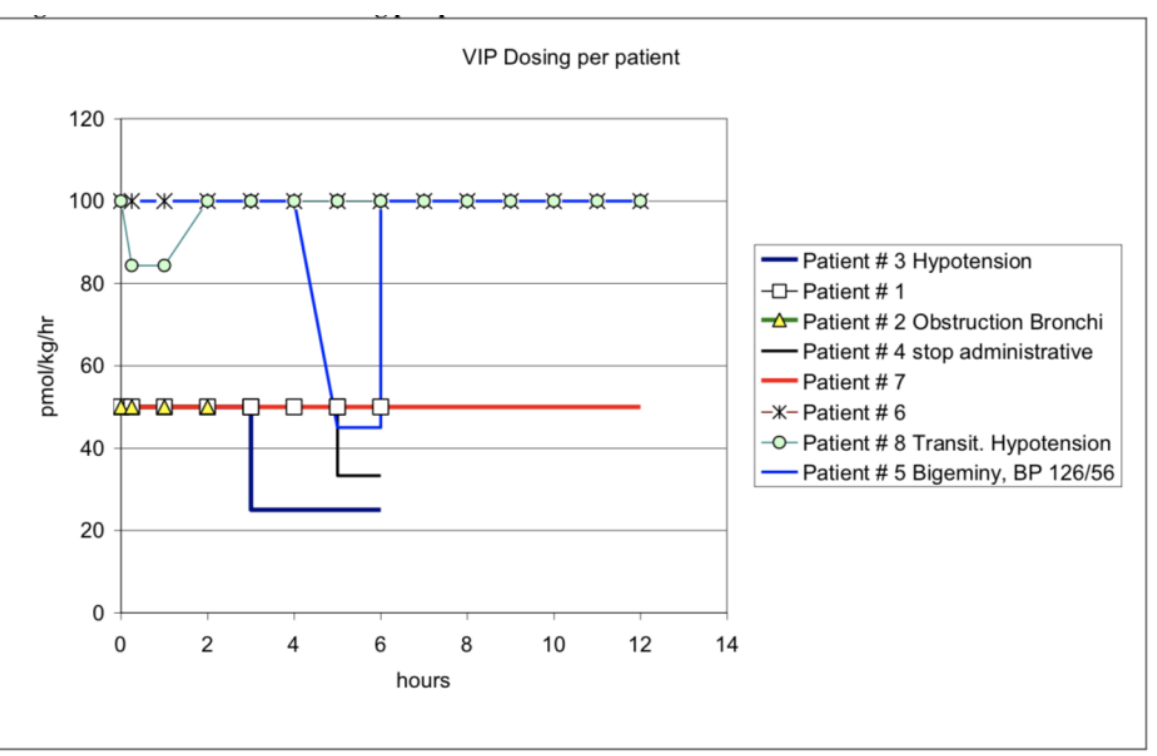

Table 1 gives an overview of the adverse events observed in these severely diseased patients. It is very likely that the adverse events of hypotension and diarrhea are attributable to Aviptadil after IV administration.

Table 1. Adverse events and outcomes following administration of IV infused VIP.

\begin{tabular}{|l|l|l|l|l|l|l|l|}
\hline $\begin{array}{l}\text { Patient } \\
\text { No. }\end{array}$ & AEs & $\begin{array}{l}\text { Description of Adverse } \\
\text { Event }\end{array}$ & Severity & Serious & Unexpected & Course & $\begin{array}{l}\text { Relationship to } \\
\text { Aviptadil }\end{array}$ \\
\hline CRF \# 1 & No & N.A. & N.A. & N.A. & N.A. & N.A. & N.A. \\
\hline CRF \# 2 & Yes & Hypoxemia, acute & Severe & Yes & No & N.A. & Unlikely \\
\hline & & Death & Severe & Yes & N.D. & N.A. & No \\
\hline CRF \# 3 & Yes & Hypotension & Mild & No & No & $\begin{array}{l}\text { disappeared after } \\
\text { intervention }\end{array}$ & Possible \\
\hline CRF \# 4 & Yes & Seizures & Severe & Yes & yes & N.D. & No \\
\hline CRF \# 5 & Yes & Bigeminy & moderate & No & No & disappeared after & intervention \\
\hline & & Diarrhea & Mild & No & Yes & Spont. disapp. & Probable \\
\hline & & Death & Extreme & Yes & No & N.A. & Probable \\
\hline CRF \# 6 & Yes & Seizures & N.A. & No & Yes & N.D. & No \\
\hline
\end{tabular}




\begin{tabular}{|l|l|l|l|l|l|l|l|}
\hline CRF \# 7 & No & N.A. & N.A. & N.A. & N.A. & N.A. & N.A. \\
\hline CRF \# 8 & Yes & Hypotension & moderate & No & No & $\begin{array}{l}\text { disappeared after } \\
\text { intervention }\end{array}$ & Probable \\
\hline & & Pneumothorax & Severe & yes & yes & $\begin{array}{l}\text { disappeared after } \\
\text { intervention }\end{array}$ & No \\
\hline
\end{tabular}




\section{Clinical Outcome}

Clinical vignettes were available for seven of eight treated patients (see supplemental material). Examination of patient vignettes reveals multiple comorbidities that are typically seen in patients who develop inpatient sepsis and progress to ARDS. Prior to treatment with VIP, patients received maximum tolerated medical therapy and were on mechanical ventilation. As seen in Table 2, patients had ARDS with moderate to poor prognosis based on $\mathrm{PaO}_{2} / \mathrm{FiO}_{2}$ ratio. All patients demonstrated improvement in oxygenation and reduced evidence of respiratory distress (see supplemental material). Seven patients were successfully extubated and discharged from the ICU. The clinical team was encouraged by improvement in the respiratory status of patient MA and believed it to be consistent with survival. However, the underlying neurologic condition in light of the patient's end of life directives caused the family to withdraw consent for further care.

Table 2: Baseline respiratory distress vs. clinical outcome

\begin{tabular}{|l|l|l|l|l|}
\hline Patient Initials & $\mathrm{PaO}_{2}$ & $\mathrm{FiO}_{2}$ & $\mathrm{PaO}_{2} / \mathrm{FiO}_{2}$ & Clinical outcome \\
\hline Patient 1 & 84 & 0.24 & $350 \mathrm{mmHg}$ & Survived \\
\hline Patient 2 & 83 & 0.8 & $104 \mathrm{mmHg}$ & Survived \\
\hline Patient 3 & 45 & 0.9 & $50 \mathrm{mmHg}$ & Survived \\
\hline Patient 4 & 115 & 0.7 & $164 \mathrm{mmHg}$ & Life support withdrawn \\
\hline Patient 5 & 59 & 0.7 & $84 \mathrm{mmHg}$ & Survived \\
\hline Patient 6 & 86 & 0.5 & $172 \mathrm{mmHg}$ & Survived \\
\hline Patient 7 & 86 & 0.88 & $98 \mathrm{mmHg}$ & Survived \\
\hline Patient 8 & Missing data & Missing data & Missing data & Survived \\
\hline
\end{tabular}

\section{Measurement of TNFa}

As an exploratory endpoint, Tissue Necrosis Factor $\alpha(\mathrm{TNF} \alpha)$ was measured in 6 of 8 patients. In five of those patients, a modest decrease in TNF $\alpha$ was seen, while patient \#6 demonstrated a substantial increase from baseline (Table 2)

Table 2: TNFa level at baseline and 24 hours post VIP infusion

TNFa Level

\begin{tabular}{|l|r|r|r|}
\hline Patient & Baseline & 24 hours & \% Change \\
\hline BA & 49.45 & 27.01 & $-45 \%$ \\
\hline GR & 25.96 & 18.73 & $-28 \%$ \\
\hline DJ & 17.69 & 12.31 & $-30 \%$ \\
\hline MA & 15.82 & 12.31 & $-22 \%$ \\
\hline LL & 13.87 & 12.31 & $-11 \%$ \\
\hline CP & 4.65 & 11.38 & $145 \%$ \\
\hline
\end{tabular}




\begin{abstract}
Discussion
This trial aimed to assess the safety of VIP as a treatment for patients with ARDS in the setting of sepsis syndrome. Such patients may or may not have evidence of other organ dysfunction. A mortality of $12.5 \%$ during the period of intensive care and of $25 \%$ at 30 days is lower than the expected mortality in sepsisrelated ARDS. Only one of the 8 patients included this study died from ARDS-related causes. The other instance of mortality was due to a massive hemorrhagic stroke rather than acute lung injury. Until recently, most studies of acute lung injury and the acute respiratory distress syndrome have reported a mortality rate of 40 to $60 \%$, although some reports suggest that mortality from this disease may be decreasing.(Bellani et al. 2016, Auriemma et al. 2020).
\end{abstract}

Although a window of 24-48 h often exists from the time sepsis/septic shock is diagnosed until severe lung and other organ injury occurs, organ injury may develop rapidly and some degree of lung injury may already be present when sepsis is first diagnosed. By limiting the study population to patients with antecedent or associated sepsis/septic shock excluding those with other risk factors for ARDS such as trauma, drug overdose, acid aspiration, and inhaled toxins, the study group was expected to be more homogeneous and well defined.

The adverse events reported above point to possible side effects of IV-infused VIP. However, the number of cases treated is insufficient and too heterogeneous to draw any conclusions regarding dose dependence or the influence of comorbidities of these adverse events. However, previous studies in which VIP was administered to humans identified similar adverse events. Krejs et al. infused VIP into healthy subjects and reported flushing, increased heart rate, and liquid stools at a dose of $400 \mathrm{pmol} / \mathrm{kh} / \mathrm{hr}$. (Krejs et al.) Kane et al. also reported watery diarrhea and subsequent metabolic acidosis within 2-6 hours of VIP infusion for 5 healthy subjects (Kane et al.). A study using inhaled VIP in patients with active sarcoidosis reported mild flatulence and loose stools as well as syncope in a small proportion of subjects. (Prasse et al.) All adverse events reported were ameliorated shortly after discontinuing treatment.

Conclusion

Intravenous Vasoactive Intestinal Peptide was generally well-tolerated at doses between 50 - 100 $\mathrm{pmole} / \mathrm{kg} / \mathrm{hr}$ in patients treated for ARDS related to sepsis. Although the study was non-randomized, survival was better than expected compared to contemporaneously reported results in this condition. The suggestion of reduced TNF $\alpha$ levels is consistent with nonclinical studies in which VIP was shown to have potent anti-cytokine effects. Based in part on these findings, synthetic VIP (aviptadil) was cleared by the US FDA in 2020 for investigational use in Critical COVID-19 with respiratory failure.

\title{
$\underline{\text { Declarations }}$
}

\section{Ethics Approval}

Human subjects were treated pursuant to IND 52,088 under a protocol approved by the Institutional Review Board of State University of New York Health Sciences Center, Stony Brook, NY.

\section{Consent for publication}

No data requiring enhanced consent for publication are included

\section{Availability of Data and Materials}

The datasets analyzed during the current study were filed with the US FDA and are available from the corresponding author on reasonable request. These data were collected in the 2000-2005 time frame at Stoneybrook University under IRB supervision. While not previously published, they were submitted to the US FDA at the time. Author JGY treated the patients and witnessed the clinical outcomes. 


\section{Authors Contributions}

Authors GJY and SS developed the protocol, treated the patients, and collected data. Authors GY and MJJ provided scientific writing. Author JCJ analyzed data and provided writing/interpretation.

\section{Competing interests}

Author JCJ is employed by NeuroRx, Inc., a pharmaceutical company that is developing VIP for human use together with Relief Therapeutics Holdings, AG. Author MCJ received compensation for medical writing.

\section{Acknowledgments}

Not applicable

\section{Authors' information}

Author JGY today is Head of Academic Pulmonary Disease at Houston Methodist Hospital. At the time these data were collected, he was research fellow to author SS.

Author SS was the discoverer of VIP in 1970 (Said and Mutt, 1970). He died in 2013 and this work was left unpublished. It was re-discovered in the process of filing IND 149,152 with the US FDA for the use of VIP in the treatment of COVID-19.

Clinical Trial Registration

This clinical trial was registered with www.clinicaltrials.gov under NCT00004494. The url of the registry record is https://www.clinicaltrials.gov/ct2/show/record/NCT00004494?term=said+vip\&draw=2\&rank=1.

\section{References:}

Auriemma CL, Zhuo H, Delucchi K, et al. Acute respiratory distress syndrome-attributable mortality in critically ill patients with sepsis. Intensive Care Med. Published online March 23, 2020. doi:10.1007/s00134-020-06010-9

Bellani G, Laffey JG, Pham T, et al. Epidemiology, Patterns of Care, and Mortality for Patients With Acute Respiratory Distress Syndrome in Intensive Care Units in 50 Countries. JAMA. 2016;315(8):788-800. doi:10.1001/jama.2016.0291

Busto R, Prieto JC, Bodega G, Zapatero J, Carrero I. Immunohistochemical localization and distribution of VIP/PACAP receptors in human lung. Peptides. 2000;21(2):265-269. doi:10.1016/s0196-9781(99)00202-8

Delgado M, Martinez C, Pozo D, et al. Vasoactive intestinal peptide (VIP) and pituitary adenylate cyclase-activation polypeptide (PACAP) protect mice from lethal endotoxemia through the inhibition of TNF-alpha and IL-6. J Immunol. 1999;162(2):1200-1205.

Feliciano L, Henning RJ. Vagal nerve stimulation releases vasoactive intestinal peptide which significantly increases coronary artery blood flow. Cardiovasc Res. 1998;40(1):45-55. doi: $10.1016 / \mathrm{s} 0008-6363(98) 00122-9$

Fraccaroli L, Grasso E, Hauk V, et al. Defects in the vasoactive intestinal peptide (VIP)/VPAC system during early stages of the placental-maternal leucocyte interaction impair the maternal 
tolerogenic response. Clin Exp Immunol. 2012;170(3):310-320. doi:10.1111/j.13652249.2012.04668.x

Frase LL, Gaffney FA, Lane LD, et al. Cardiovascular effects of vasoactive intestinal peptide in healthy subjects. Am J Cardiol. 1987;60(16):1356-1361. doi:10.1016/0002-9149(87)90619-9

Ganea D, Hooper KM, Kong W. The neuropeptide vasoactive intestinal peptide: direct effects on immune cells and involvement in inflammatory and autoimmune diseases. Acta Physiol (Oxf). 2015;213(2):442-452. doi:10.1111/apha.12427

Hernanz A, Tato E, De la Fuente M, de Miguel E, Arnalich F. Differential effects of gastrinreleasing peptide, neuropeptide $Y$, somatostatin and vasoactive intestinal peptide on interleukin-1 beta, interleukin- 6 and tumor necrosis factor-alpha production by whole blood cells from healthy young and old subjects. J Neuroimmunol. 1996;71(1-2):25-30. doi:10.1016/s0165$\underline{\text { 5728(96)00118-X }}$

Kane MG, O'Dorisio TM, Krejs GJ. Production of secretory diarrhea by intravenous infusion of vasoactive intestinal polypeptide. N Engl J Med. 1983;309(24):1482-1485. doi:10.1056/NEJM198312153092403

Ke L-Q, Wang F-M, Luo Y-C. [Effects of vasoactive intestinal peptide on airway inflammation and Th17/Treg balance in asthmatic mice]. Zhongguo Dang Dai Er Ke Za Zhi. 2017;19(6):699704.

Krejs GJ, Fordtran JS, Fahrenkrug J, et al. Effect of VIP infusion in water and ion transport in the human jejunum. Gastroenterology. 1980;78(4):722-727.

Li L, Luo ZQ, Zhou M, et. al. Effect of vasoactive intestinal peptide on pulmonary surfactants phospholipid synthesis in lung explants. Acta Pharmacol Sin 2004;25:1652-1658.

Martínez C, Delgado M, Pozo D, et al. Vasoactive intestinal peptide and pituitary adenylate cyclase-activating polypeptide modulate endotoxin-induced IL-6 production by murine peritoneal macrophages. J Leukoc Biol. 1998;63(5):591-601. doi:10.1002/jlb.63.5.591

Murray JF, Matthay MA, Luce JM \& Flick MR. An expanded definition of the adult respiratory distress syndrome. Am Rev Resp Dis 1988;138: 720-723.

Nicholas TE: Pulmonary surfactant: no mere paint on the alveolar wall. Respirology 1996, $1: 247-257$.

Onoue S, Ohmori Y, Endo K, Yamada S, Kimura R, Yajima T. Vasoactive intestinal peptide and pituitary adenylate cyclase-activating polypeptide attenuate the cigarette smoke extract-induced apoptotic death of rat alveolar L2 cells. Eur J Biochem. 2004;271(9):1757-1767.

doi:10.1111/j.1432-1033.2004.04086.x 
Power RF, Bishop AE, Wharton J, et al. Anatomical distribution of vasoactive intestinal peptide binding sites in peripheral tissues investigated by in vitro autoradiography. Ann N Y Acad Sci. 1988;527:314-325. doi:10.1111/j.1749-6632.1988.tb26989.x

Prasse A, Zissel G, Lützen N, et al. Inhaled vasoactive intestinal peptide exerts immunoregulatory effects in sarcoidosis. Am J Respir Crit Care Med. 2010;182(4):540-548. doi: $10.1164 / \mathrm{rccm} .200909-14510 \mathrm{C}$

Said SI, Mutt V. Potent peripheral and splanchnic vasodilator peptide from normal gut. Nature. 1970; 225: 863-864.

Ran W-Z, Dong L, Tang C-Y, et al. Vasoactive intestinal peptide suppresses macrophagemediated inflammation by downregulating interleukin-17A expression via PKA- and PKCdependent pathways. Int J Exp Pathol. 2015;96(4):269-275. doi:10.1111/iep.12130

Smitherman TC, Popma JJ, Said SI, Krejs GJ, Dehmer GJ. Coronary hemodynamic effects of intravenous vasoactive intestinal peptide in humans. American Journal of Physiology-Heart and Circulatory Physiology. 1989;257(4):H1254-H1262. doi:10.1152/ajpheart.1989.257.4.H1254 